\title{
A Systematic Review of the History, Cause, and Treatment of Persistent Depressive Disorder
}

\author{
Jiaren $\mathrm{Lu}^{1, *}$

\begin{abstract}
${ }^{1}$ Humanities and Social Sciences, College of Fine Arts, University of Massachusetts Lowell, Lowell, Massachusetts, United States

*Corresponding author. Email: Jiaren_Lu@student.uml.edu
\end{abstract}

\begin{abstract}
Persistent depressive disorder (PDD) is a new classification in the DSM-5 that includes dysthymia, chronic major depressive disorder, recurrent major depressive disorder, and dual depression. Although a new concept, PDD is already a common mood disorder. However, information about the disease is limited. This review provides an overview of the history of PDD, clarifies the definition of persistent depression, and explores some of the controversies surrounding PDD. The results showed that there are many possible causes, including family and social factors. This review also includes new advances in therapies currently approved by major scientific bodies, including psychotherapy and pharmacotherapy, and provides a new perspective for future treatment and recurrence prevention. There is little research on PDD, specifically in biology, and researchers could put more effort into this area.
\end{abstract}

Keywords: Persistent depressive disorder, Dysthymia, Psychological treatment, Combination therapy

\section{INTRODUCTION}

On the Internet, people often use "depression" to describe how "sad" they feel. However, in the clinical sense, depression is a serious mental illness. It affects millions of people every year. Depression has become a major problem in society. Persistent depressive disorder (PDD) or Dysthymia (DST) described here is a part of depression that is usually characterized by mild but PDD. The illness is usually less severe than a major depressive episode but can last for years. It will cause a heavy burden on individuals, families, and even the whole society [1]. Many patients suffer from the condition for so long that some think it is part of their personality and are dominated by pessimism.

PDD is a new concept proposed by psychologists of the American Psychiatric Association in the Diagnostic and Statistical Manual (DSM -5), which includes various symptoms such as chronic major depression (CMDD) and Dysthymia [2]. It's important to note that dysthymia and major depression can occur together. This condition is often referred to as double depression (DD). According to a large survey by the National Comorbidity Survey (NCS), $2.7 \%$ of Americans have suffered from Persistent depressive disorder at some point in their lives, and $4.1 \%$ have experienced depression or dysthymia throughout their lives. However, only about $3.4 \%$ of Americans get the correct diagnosis [3]. According to Murphy and Byrne [4], the prevalence of PDD in Australia is 4.6\%. They concluded that PDD seems to be very common in the community. In clinical Settings, persistent depressive disorder is more common. After investigating psychiatric outpatients and private clinics, researchers found that patients with persistent depressive disorder accounted for about $50 \%$ of patients with depression [5]. The rate of chronic depression in women is almost twice that of men [6].

Given that there are many differences in the variables associated with the cause of depression, there are also different forms of PDD. Among them, PDD is especially easy to be ignored by people. Because it's milder than other depressions and can be hard to distinguish. Therefore, it sets the tone for further research and future treatment. This review aims to summarize the classification, causes and treatment, and discuss and refute the controversy surrounding the concept of PDD.

\section{METHOD}

During this study, a systematic search was conducted using the University of Massachusetts Lowell libraries and Google Scholar. The search keywords mainly focus on "PDD" or "persistent depressive disorder" or "Dysthymia". The selected article topics were examined 
for relevant research using DSM-5 as the main criteria. In addition, some screening has been carried out. The selection criteria are as follows: (1) articles must be published in full, in English, and have been peerreviewed. (2) The article must be original. (3) The author must use scientific and reliable tools to measure and diagnose the persistent depressive disorder. (4) The participants of the article should be human; if not, the article will be excluded.

In total, 17 articles were included in this paper.

\section{RESULTS}

Taken together, this review includes all aspects of persistent depression. Next, we will introduce each of them.

\subsection{History}

The problem of Persistent depressive mood has been known since ancient Greek times. In the 19th century, Kahlbaum provided the first modern description of dysthymia, which he distinguished from normal fluctuating emotions [7]. Later, DSM-II defined chronic depression as a personality disorder, but Akiskal considered it as a mood disorder in his research [8]. This provides an important theoretical basis for the future introduction of DST as a discrete classification of affective disorders into DSM-III. Dysthymia was subsequently introduced into the DSM-III and ICD-10 as a diagnostic criterion for chronic depression.

\subsection{Modern definition}

The modern definition of dysthymia (DST) is defined according to DSM-IV-TR (Diagnostic and Statistical Manual of Mental Disorders, 4th Edition) and ICD-10 (International Classification, 10th Edition), where depressive mood must have lasted for at least 2 years and be less severe than a major depressive disorder (MDD) [9]. When determining whether a patient has DST, medical advocates need to determine whether the patient has had an episode of MDD in the previous two years. As mentioned earlier, DST can occur at the same time as MDD, a condition commonly known as double depression (DD). Chronic major depression (CMDD) is diagnosed when MDD lasts more than two years. In the DSM-5, these conditions are grouped under the category of persistent depressive disorder (PDD). According to the manual, PDD consists of four conditions (1) only dysthymia; (2) CMDD; (3) INTERMITTENT MDD, current episode; (4) Intermittent MDD, no current episode [2]. Therefore, persistent depression is commonly used in the current literature to replace the above four conditions.

\subsection{Etiology}

In general, the causes of Persistent Depressive Disorder include mental and physical aspects. Next, we will introduce each of them.

\subsubsection{Biology}

There is little research on the biology of Persistent Depressive Disorder. While the term "dysthymia" could be used instead of searching, most of the studies are outdated. There is a lack of research in this area.

\subsubsection{Genetics}

However, available data and statistics suggest that genetic factors may contribute to Persistent Depressive Disorder. Based on Mondimore's research [9], they found that Persistent Depressive Disorder runs in families. It is especially common in families with prepubertal traits. For major depression, which is often compared to Persistent Depressive Disorder, there is also a strong family connection for major depression, but Persistent Depressive Disorder is much stronger [10].

\subsubsection{Family factors}

\subsubsection{Physical Abuses}

A history of childhood abuse, including physical, sexual, and emotional abuse, can lead to Persistent Depressive Disorder. Abuse is often cited as a cause of depression. It includes physical abuse, psychological abuse, and neglect. Abusive behavior can damage the patient's physical and mental health, resulting in mental impairment. In addition, maltreatment that occurs in childhood has long-term effects. Regardless of treatment, patients with a history of childhood maltreatment are more likely to develop chronic diseases and are more difficult to treat [11].

\subsubsection{Sexual abuse}

Sexual abuse is the physical assault of a person with violence or threats to satisfy sexual desire. According to Cheasty's survey records, sexual abuse in childhood is one of the predictors of Persistent Depressive Disorder in adulthood [12].

\subsubsection{Psychological \& Neglect}

It's not just physical abuse, it's psychological abuse. Parental abuse and neglect of children is a form of psychological abuse. Psychological abuse may sometimes affect the mental health of the victim more than physical abuse [13]. People who suffer from emotional abuse and neglect are more likely to develop personalities with high sensitivity and low self-esteem, leading to Persistent Depressive Disorder [11]. 


\subsection{Clinical treatment}

Many clinicians intuitively believe that because Persistent Depressive Disorder is chronic and strongly influenced by environmental factors, patients may be in greater need of psychotherapy. However, it may be the opposite. Based on Cijpers [14], the effect of psychotherapy alone on Persistent Depressive Disorder is small, and the effect of psychotherapy may be overestimated. According to research, psychotherapy is effective for chronic depression and dysthymia, but may not be as effective as medication (especially SSRIs) [15]. In any case, antidepressants and psychotherapy are two ways to treat Persistent Depressive Disorder. Doctors can use them alone or in combination, and treatment plans should be tailored to the individual patient.

\subsubsection{Pharmacotherapy}

In a study of Kriston's meta-analysis [16], several medications can be used to treat Persistent Depressive Disorder, such as Moclobemide, AmisulPride, and Sertraline, which are significantly better than placebo. Patients need to communicate with their doctors carefully and not hide anything. Doctors also need to be particularly vigilant and careful and complete the patient's medical history.

\subsubsection{Psychotherapy}

Although psychotherapy is not as effective as medication, it is an essential part of the treatment of Persistent Depressive Disorder. Psychotherapy and medication have been compared, but it is important to note that, as technology has evolved, cognitive behavioral therapy (CBT) has not been included in this comparison. The Cognitive Behavioral Analysis System (CBASP) for psychotherapy is the only psychotherapy developed specifically for PDD treatment [17]. Doctors help patients with interpersonal therapy (IPT) and psychological therapy's Cognitive Behavioral Analysis system (CBASP). Cognitive-behavioral therapy is by far the most widely tested psychotherapy for Persistent Depressive Disorder. In Furukawa's paper, he and his colleagues compared CBASP, antidepressant therapy, and their combinations. They want to know which is the most effective. They concluded that the combination therapy had a significant advantage over both in terms of efficacy and acceptability. Cuijpers also pointed out in his analysis that psychotherapy had a significant effect on Persistent Depressive Disorder compared to the control group [15]. Longer treatment was associated with better outcomes with more sessions. Regarding the benefits of targeted therapy, a study has shown that CBASP is sometimes more effective than common drug therapy in patients with early-onset Persistent Depressive Disorder who are not taking medication, especially for those with childhood abuse and social anxiety disorder [18].

\subsubsection{Combination Therapy}

From the above, patients with PDD will likely gain more acute benefits from combination therapy [14]. On average, the combination of CBASP and medication showed significant benefits in patients with PDD. In another study of patients with Persistent Depressive Disorder, the combination significantly improved quality of life [19]. Most patients prefer combination therapy to monotherapy. However, this needs to be more costeffective. As a result, patients are likely to be unable to afford the heavy debt. On the other hand, every person's situation is different, and it is difficult for doctors to guarantee that a combination of treatments will work.

\subsection{Some existing problems}

Although there are existing medical methods to treat Persistent Depressive Disorder, in clinical practice, doctors are still unable to do anything about the low success rate of treating Persistent Depressive Disorder and the high recurrence rate after treatment. Several factors may limit the success of treatment for Persistent Depressive Disorder [18]: First, treatment delays patients often do not seek treatment for years after onset because of fear of discrimination. Second, not realizing it, most PDD people see pessimism and irritability as part of their personality that cannot be treated. Third, long periods of treatment - Because of the characteristics of Persistent Depressive Disorder, even with adequate treatment, some patients who appear to have been cured become inactive later in treatment, leading to relapse. Finally, resistance to treatment - PDD brings pessimistic thoughts that lead patients to negative treatment. They think, "It's not going to work anyway." Therefore, therapists should tell patients from the outset that PDD is a mood disorder and not an incurable one. At the same time, the correct way is to plan the course of treatment, actively follow up on the patient's situation, and give the patient encouragement and positive feedback.

\subsection{Controversy}

The introduction of DST into DSM-III has been controversial since many believe that symptoms of a bad mood can often be traced back to the shadow of childhood or adolescence [19]. In particular, a new concept in the DSM-5, persistent depression, has come under fierce attack from critics. They argue that this diagnosis should be eliminated because there is currently a lack of theoretical basis and supporting evidence for merging CMDD and DST [20]. The report from Klein showed that relatives of people with persistent depression were more likely to develop it. Almost all patients with dysthymia will eventually develop into severe depression due to family reasons [21]. As a result, critics prefer PDD or DST as a personality disorder. 
However, most critics ignore much of the evidence that establishes PDD. The response of patients with dysthymic depression drugs was significantly better than that of placebo [22]. So, despite the connection to personality disorders, this is evidence that dysthymia is a mood disorder. From a medical point of view, the presence of PDD can help people better define depression symptoms are cognitive problems. In contrast, other depression is more manifested as motor and neurological symptoms [23]. Although it can be difficult to distinguish PDD from other forms of depression, many people with depression present with chronic symptoms.

\section{DISCUSSION}

Taken together, As described in this paper, many factors can lead to PDD, including family, social, environmental, and biological factors. Therefore, it is unlikely that every patient will be cured using the same approach. The treatment of PDD requires unique treatments tailored to each patient. Doctors need to actively communicate with their patients and assess whether the most appropriate treatment option is available. The difficulties commonly encountered in clinical diagnosis have been highlighted several times in this review. One key to treating persistent diseases is patient and physician persistence. The same is true of PDD. Patients often need to undergo a long period of treatment. It is not surprising, then, that patients would question the diagnosis when their treatment failed.

What needs to be improved so far is: Most research on depression has focused on episodic depression. This has led to a lack of research on depression. One of the most important factors that make Persistent Depressive Disorder clinically difficult is that it needs to be examined by doctors with extensive experience. Unfortunately, research and development into the treatment of Persistent Depressive Disorder have been hampered by ambiguities in definitions and diagnostic criteria, as well as underestimates of its severity.

At the same time, psychologists need to do more research into treatment outcomes and procedures. This can determine which patients are benefiting from which part of which treatment. This can help doctors determine the best combination of psychotherapy and drug interventions. Modular treatment tailored to individual patients' therapeutic factors may help reduce the personal and social burden of persistent depressive disorder.

When the treatment is complete, and the patient feels better, the patient should not take it lightly. They also need to take care to prevent relapses. The clinical manifestation of Persistent Depressive Disorder is a chronic disease with a high recurrence rate. There is evidence of increased treatment resistance and suicide risk in patients with disease relapse, as well as antidepressant drug resistance [24]. As a result, patients with Persistent Depressive Disorder are often recommended for long-term treatment. Patients should not stop taking their medication without talking to their doctor. For patients with multiple relapses, especially high-risk patients, not only drug therapy but also longterm psychological therapy (such as CBT, IPT, etc.). This combination therapy improves patients' frequent recurrence [25]. The patients themselves should reflect on why they are ill, make changes in time, and find ways to cope with stress and improve self-esteem. They should make sure they get enough sleep, eat healthy food, and exercise regularly. When in trouble, contact family and friends and regular medical check-ups.

Persistent depression is a particularly complex classification of mental disorders, including a variety of different conditions. It's very individual-specific. This review summarizes the most relevant and recent articles in the field in terms of definitions, identification in clinical settings, and how to treat them. This can provide the reader with a general overview of the condition and help doctors diagnose and treat it better. More clinical trials of PDD are urgently needed, especially in brain function and neurology. It is also hoped that more attention will be paid to improving patients' quality of life with persistent depression, as patients often see these goals as the main purpose of their treatment.

\section{CONCLUSION}

Overall, PDD can be caused by many factors, including interpersonal, family, social, emotional, environmental, and individual factors. It can significantly reduce a person's quality of life and daily performance. PDD is a particularly complex disease because it's very individual-specific. Therefore, it is unlikely to be universally successful without using different treatment methods. The treatment of persistent depression requires a combination of psychotherapy and medication, such as integrating medication into CBASP. As mentioned above, combination therapies are more effective than traditional therapy because each patient gets a different treatment tailored to their condition. Patients can give doctors feedback on their own experiences. Maybe it will produce an algorithm. This algorithm can help doctors rank psychological treatments and drug interventions to help patients get the best combination. More clinical trials of PDD are urgently needed, especially in the areas of brain neuroscience and physiology. This can lead to a burden on the field of depression. Meanwhile, it is hoped that more people will pay attention to improving PDD patients' quality of life, accommodate and help them.

\section{REFERENCES}

[1] Greenberg PE, Kessler RC, Birnbaum HG, Leong SA, Lowe SW, Berglund PA, Corey-Lisle PK. The economic burden of depression in the United States: 
how did it change between 1990 and 2000? J Clin Psychiatry. 2003 Dec;64(12):1465-75. doi: 10.4088/jcp.v64n1211. PMID: 14728109.

[2] American Psychiatric Association. Diagnostic and statistical manual of mental disorders (5th ed.). Washington, DC: Author; 2013.

[3] National Comorbidity http://www.hcp.med.harvard.edu/ncs.

Survey. June 6, 2014.

[4] Murphy, J. A., \& Byrne, G. J. (2012). Prevalence and correlates of the proposed DSM-5 diagnosis of Chronic Depressive Disorder. Journal of Affective Disorders, 139(2), 172-180. https://doiorg.umasslowell.idm.oclc.org/10.1016/j.jad.2012.0 1.033

[5] Benazzi, F. (1998). Chronic depression: A case series of 203 outpatients treated at a private practice. Journal of Psychiatry \& Neuroscience, 23(1), 51-55.

[6] Ildirli S, Şair YB, Dereboy F. Persistent Depression as a Novel Diagnostic Category: Results from the Menderes Depression Study. Noro Psikiyatr Ars. 2015 Dec;52(4):359-366. doi: 10.5152/npa.2015.7589. Epub 2015 Dec 1. PMID: 28360740 ; PMCID: PMC5353108.

[7] Freeman HL. Historical and nosological aspects of dysthymia. Acta Psychiatr Scand Suppl. 1994;383:7-11. doi: 10.1111/j.16000447.1994.tb05877.x. PMID: 7942068

[8] Akiskal HS, Bitar AH, Puzantian VR, Rosenthal TL, Walker PW. The Nosological Status of Neurotic Depression: A Prospective Three- to Four-Year Follow-up Examination in Light of the PrimarySecondary and Unipolar-Bipolar Dichotomies. Arch Gen Psychiatry. 1978;35(6):756-766. doi:10.1001/archpsyc.1978.01770300098011

[9] Mondimore FM, Zandi PP, Mackinnon DF, McInnis MG, Miller EB, Crowe RP, Scheftner WA, Marta DH, Weissman MM, Levinson DF, Murphy-Ebenez KP, Depaulo JR Jr, Potash JB. Familial aggregation of illness chronicity in recurrent, early-onset major depression pedigrees. Am J Psychiatry. 2006 Sep;163(9):1554-60. doi: 10.1176/ajp.2006.163.9.1554. PMID: 16946180.

[10] Klein DN, Riso LP, Donaldson SK, et al. Family Study of Early-Onset Dysthymia: Mood and Personality Disorders in Relatives of Outpatients With Dysthymia and Episodic Major Depression and Normal Controls. Arch Gen Psychiatry. 1995; 52(6):487-496.

doi:10.1001/archpsyc.1995.03950180073010
[11] Brown GW, Craig TK, Harris TO. Parental maltreatment and proximal risk factors using the Childhood Experience of Care \& Abuse (CECA) instrument: a life-course study of adult chronic depression - 5. J Affect Disord. 2008 Oct;110(3):222-33. doi: 10.1016/j.jad.2008.01.016. Epub 2008 Mar 11. PMID: 18334270.

[12] Cheasty, M., Clare, A., \& Collins, C. (2002). Child sexual abuse - a predictor of Persistent Depressive Disorder in adult rape and sexual assault victims. Journal of Mental Health, 11, 79 - 84.

[13] Nanni V, Uher R, Danese A. Childhood maltreatment predicts unfavorable course of illness and treatment outcome in depression: a metaanalysis. Am J Psychiatry. 2012 Feb;169(2):141-51. doi: 10.1176/appi.ajp.2011.11020335. Erratum in: Am J Psychiatry. 2012 Apr;169(4):439. PMID: 22420036.

[14] Cuijpers P, Andersson G, Donker T, van Straten A. Psychological treatment of depression: results of a series of meta-analyses. Nord J Psychiatry. 2011 Dec;65(6):354-64.

doi: 10.3109/08039488.2011.596570. Epub 2011 Jul 20. PMID: 21770842

[15] Cuijpers P, van Straten A, Schuurmans J, van Oppen P, Hollon SD, Andersson G. Psychotherapy for chronic major depression and dysthymia: a metaanalysis. Clin Psychol Rev. 2010 Feb;30(1):51-62. doi: 10.1016/j.cpr.2009.09.003. PMID: 19781837.

[16] Kriston L, von Wolff A, Westphal A, Hölzel LP, Härter M. Efficacy and acceptability of acute treatments for persistent depressive disorder: a network meta-analysis. Depress Anxiety. 2014 Aug;31(8):621-30. doi: 10.1002/da.22236. Epub 2014 Jan 21. PMID: 24448972.

[17] Furukawa T, A, Efthimiou O, Weitz E, S, Cipriani A, Keller M, B, Kocsis J, H, Klein D, N, Michalak J, Salanti G, Cuijpers P, Schramm E: CognitiveBehavioral Analysis System of Psychotherapy, Drug, or Their Combination for Persistent Depressive Disorder: Personalizing the Treatment Choice Using Individual Participant Data Network Metaregression. Psychother Psychosom 2018;87:140-153. doi: 10.1159/000489227

[18] Schramm, E., Klein, D. N., Elsaesser, M., Furukawa, T. A., \& Domschke, K. (2020). Review of dysthymia and persistent depressive disorder: history, correlates, and clinical implications. The Lancet Psychiatry, 7(9), 801-812. https://doiorg.umasslowell.idm.oclc.org/10.1016/S22150366(20)30099-7 
[19] Kocsis JH, Frances AJ. A critical discussion of DSM-III dysthymic disorder. Am J Psychiatry. 1987 Dec;144(12):1534-42. doi: 10.1176/ajp.144.12.1534. PMID: 3318511

[20] Parker G, Malhi GS. Persistent depression: should such a DSM-5 diagnostic category persist? Can J Psychiatry. 2019; 64(3):177-179.

[21] Klein DN, Shankman SA, Lewinsohn PM, Rohde P, Seeley JR. Family study of chronic depression in a community sample of young adults. Am J Psychiatry. 2004 Apr;161(4):646-53. doi: 10.1176/appi.ajp.161.4.646. PMID: 15056510.

[22] von Wolff A, Hölzel LP, Westphal A, Härter M, Kriston L. Selective serotonin reuptake inhibitors and tricyclic antidepressants in the acute treatment of chronic depression and dysthymia: a systematic review and meta-analysis. J Affect Disord. 2013 Jan 10;144(1-2):7-15. doi: 10.1016/j.jad.2012.06.007. Epub 2012 Sep 7. PMID: 22963896.

[23] Klein DN, Kocsis JH, McCullough JP, Holzer CE 3rd, Hirschfeld RM, Keller MB. Symptomatology in dysthymic and major depressive disorder. Psychiatr Clin North Am. 1996 Mar;19(1):41-53. doi: 10.1016/s0193-953x(05)70272-0. PMID: 8677219.

[24] Keller MB, Lavori PW, Mueller TI, Endicott J, Coryell W, Hirschfeld RM, Shea T. Time to recovery, chronicity, and levels of psychopathology in major depression. A 5-year prospective follow-up of 431 subjects. Arch Gen Psychiatry. 1992 Oct;49(10):809-16.

doi: 10.1001/archpsyc.1992.01820100053010. PMID: 1417434.

[25] Thase, Michael E. (2006). Preventing Relapse and Recurrence of Depression: A Brief Review of Therapeutic Options. CNS Spectrums, 11(S15), 1221. doi: $10.1017 / \mathrm{s} 1092852900015212$ 